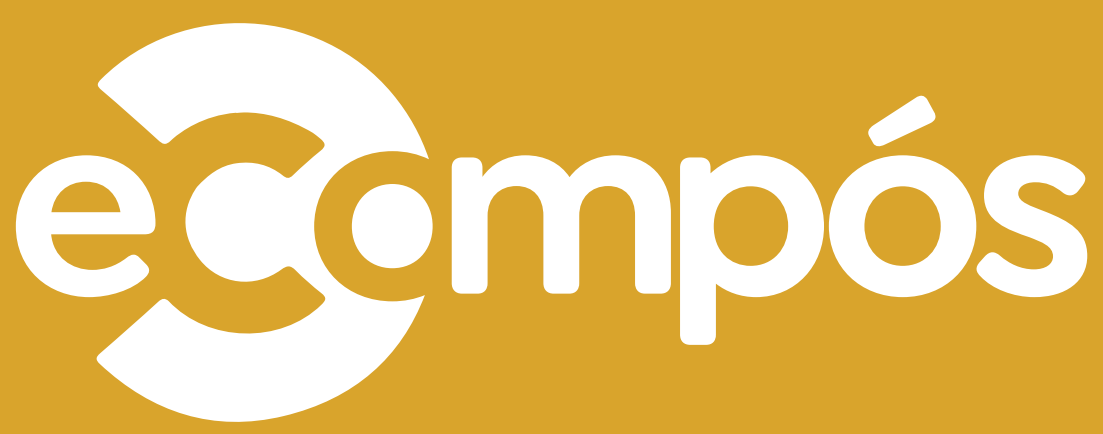

Revista da Associação Nacional dos Programas de Pós-Graduação em Comunicação

ISSN 1808-2599, v. 24, jan-dez,

publicação contínua, 2021, p. 1-20.

doi.org/10.30962/ec.2287

\title{
Midiatização Profunda, Plataformas e Logjects
}

\section{LIRÁUCIO GIRARDI JR.}

Universidade Municipal de São Caetano do Sul, São Paulo, Brasil Faculdade Cásper Líbero, São Paulo, São Paulo, Brasil

\section{ID 2287}

Recebido em

22/08/2020

Aceito em

06/01/2021 


\section{/ resumo}

Neste artigo, procuramos mostrar como a noção de midiatização profunda, proposta por Couldry e Hepp (2017), pode ser uma referência importante para os estudos de comunicação, se levarmos em conta a natureza das plataformas, dos objetos digitais e as formas culturais assumidas por esses novos ambientes comunicacionais. Entre elas, podemos destacar a chamada cultura da busca, a cultura algorítmica ou a cultura orientada por dados (CHENEY-LIPPOLD, 2017 ANDERSEN, 2018, GILLESPIE, 2018b). Avançando um pouco mais nessas reflexões, procuramos apontar, ainda, como a particularidade dos objetos digitais, no caso, os logjects, objetos codificados de registro e de compartilhamento de dados (DODGE; KITCHIN, 2009) podem nos ajudar na construção de hipóteses de investigação sobre o aprofundamento das formas de midiatização contemporâneas.

Palavras-chave: Midiatização profunda. Plataformas. Algoritmos. Cultura da busca. Logjects.

\section{Mediatización Profunda, Plataformas e Logjects}

En este artículo tratamos de mostrar cómo la noción de mediatización profunda, propuesta por Couldry y Hepp (2017), puede ser una referencia para los estudios de comunicación si tenemos en cuenta la naturaleza de las plataformas, los objetos digitales e las formas culturales asumidas por estos nuevos entornos comunicacionales. Entre ellos, podemos destacar la cultura de búsqueda, la cultura algorítmica o la cultura basada en datos (CHENEY-LIPPOLD, 2017 ANDERSEN, 2018, GILLESPIE, 2018b). Avanzando en estas reflexiones, tratamos de señalar cómo la particularidad de los objetos digitales, en este caso, los objetos codificados $y$ en red (logjects) (DODGE; KITCHIN, 2009), nos puede ayudar en la construcción de hipótesis de investigación sobre la profundización de las formas contemporáneas de mediatización.

Palabras clave: Mediatización profunda.

Plataformas. Algoritmos. Cultura de búsqueda. Logjects.

\section{Deep Mediatization, Platforms and Logjects}

In this article, we aim to understand how the notion of deep mediatization, proposed by Couldry and Hepp (2017), can be an important reference for communication studies, taking into account the nature of platforms and digital objects. In this direction, it was also necessary to reflect on the cultural forms assumed by these new communicational environments. Among them, we can highlight the culture of search, the algorithmic culture or the data-driven culture (CHENEY-LIPPOLD, 2017 ANDERSEN, 2018, GILLESPIE, 2018b). Advancing in these reflections, we try to point out how the particularity of digital objects, in this case, coded and networked objects (logjects) (DODGE; KITCHIN, 2009), can help us to construct hypotheses of investigation to understand the deep contemporary forms of mediatization.

Keywords: Deep mediatization. Platforms. Algorithms. Search culture. Logjects. 


\section{/autor}

\section{Liráucio
GIRARDI JÚNIOR}

Doutor em Sociologia pelo Programa de Pós-Graduação

em Sociologia da Universidade de São Paulo - USP.

Professor e Pesquisador do Mestrado Profissional

em Inovação na Comunicação de Interesse Público

- USCS e Mestrado Acadêmico em "Comunicação na

Contemporaneidade" - Faculdade Cásper Líbero.

Universidade Municipal de São Caetano do Sul, São

Paulo, Brasil.

Faculdade Cásper Líbero, São Paulo, São Paulo, Brasil.

E-mail: lira.sociologia@gmail.com

\section{ORCID}

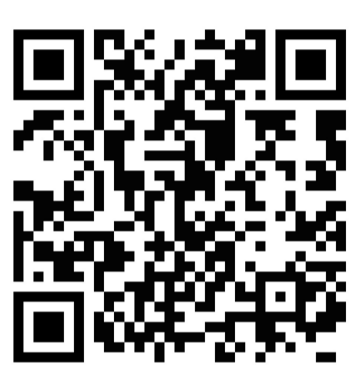




\section{Introdução}

Podemos considerar a tradução para o português, em 2020, de A Construção Mediada da Realidade, pela editora Unisinos, um momento oportuno para a retomada de um processo de interlocução com os autores sobre os possiveis desdobramentos de suas abordagens.

Portanto, pretendemos identificar, neste artigo, como as plataformas, os objetos digitais e a complexidade dos ambientes comunicacionais contemporâneos podem gerar uma busca pelo entendimento daquilo que Couldry e Hepp chamaram de midiatização profunda.

Gostaríamos de ressaltar que não pretendemos discutir aqui a importância da noção de midiatização como um elemento unificador das pesquisas na área de comunicação e nem explorar as divergências em torno de sua definição, ou mesmo, de sua relevância como processos de articulação: midiatização fraca ou forte; sob uma perspectiva institucional ou socioconstrutivista; como processos interacionais de referência ou de mútua determinação entre dispositivos, processos comunicacionais e processos sociais; como bios ou, ainda, como um conceito-ônibus (capaz de abarcar quase tudo) etc.

No Brasil, uma análise crítica sobre os seus usos pode ser encontrada nos trabalhos organizados por Janotti Jr., Mattos, Jacks (2012) e Ferreira et al. (2018), artigos e livros publicados por Sodré (2002), Braga (2006), Fausto Neto (2006). Ferreira (2007), Rüdiger (2015), Gomes (2016), Carvalho (2016), Martino (2019) entre outros. Temos a visão "semioantropológica" de Véron (2014), a análise da pesquisadora portuguesa Figueiras (2017), e as sistematizações e críticas levantadas por Couldry (2008), Couldry e Hepp (2013), Hepp (2014), Ampuja, Koivisto, Väliverronen (2014), Hepp, Hjarvard, Lundby (2015) e Hjarvard (2012, 2015) quanto à sua definição e seus usos. Entre as abordagens que procuraram pensar a relação entre midiatização, mídias digitais e plataformização podemos destacar Finnemann (2011), Mintz (2019), Grohmann e Mauro (2015) e Grohmann (2020).

A complexidade desse debate tem mostrado como a noção de midiatização pode ser pensada de diferentes formas, ocorrendo em diversos níveis e podendo ser considerada, até mesmo, insignificante sob certas condições sociais, culturais e históricas. Neste artigo, particularmente, acompanhamos uma orientação destacada por Figueiras:

Os media-como-instituição e os media-como-ambiente perpassam todas as áreas da vida, logo, todas as áreas científicas, pelo que a ausência da análise desses processos significa que há lacunas evidentes no conhecimento que essas variadas áreas de saber produzem sobre si próprias, sendo a mediatização um conceito-chave que pode contribuir para retirar os media das margens das demais ciências sociais e humanas (FIGUEIRAS, 2017 p. 121).

É a partir desta linha de abordagem que pretendemos avançar na direção daquilo que Couldry e Hepp chamam de novas configurações comunicacionais, presentes no processo de midiatização profunda, e, a partir delas, procurarmos entender qual seria o lugar das plataformas e dos objetos digitais na construção de formas culturais que demarcam esse aprofundamento.

Para isso, em primeiro lugar, precisamos levar em consideração as observações de Gunkel (2009) sobre a natureza de objetos comunicacionais muito particulares que se apresentam nesse ap rofundamento: os dispositivos e os objetos computacionais. Entendemos que o processo de aprofundamento poderia estar relacionado à natureza particular desses dispositivos e objetos em meio aos quais se formam ambientes comunicacionais marcados por formas culturais específicas: a cultura da busca e a cultura do algoritmo integrada a complexos mecanismos de registro, arquivamento, indexação e acesso a bancos de dados, tal como proposta por Jack Andersen (2018)'.

1 A posição deste autor é sustentada, ainda, pelas análises de Striphas (2015), Cheney-Lippold (2011), Gillespie (2018a, 2018b), autores que tivemos oportunidade de discutir de modo mais aprofundado em outro artigo (GIRARDI JR., 2017) 
Todo esse processo tende a se tornar, ainda, mais complexo se trouxermos para o debate a existência de certos objetos digitais que são fundamentais na existência dos meios de comunicação contemporâneos: os chamados logjects (DODGE; KITCHIN, 2009, 2011; BROTAS, 2017). Em nossa abordagem, eles passam a representar aquilo que Couldry e Hepp (2017) chamaram de forças de moldagem de uma midiatização profunda.

Os logjects são certos tipos de objetos digitais, que possuem software "embarcado", são programáveis e realizam processos de registro de informações da relação entre eles e os seus usuários, compartilhando essas informações em rede com outros logjects. Além disso, são capazes de reconhecer esses padrões de uso, orientar ou tomar certos tipos de "decisões" como: ligar luzes, fazer um pedido por aplicativo, pagar contas, organizar e eliminar e-mails, recomendar leituras, lembrar de eventos, organizar textos, reconhecer rostos etc.

A integração de aplicativos (apps) aos dispositivos digitais móveis (smartphones), aos wearables (objetos digitais vestíveis) e aos assistentes pessoais, são os exemplos mais visíveis desses tipos de objetos. Eles são tipos particulares de agentes, capazes de assumir formas diversas, podendo ser vistos, ao mesmo tempo, como códigos, textos, máquinas, atores e ambientes (JOHNSON, 2001). Todos eles, de variadas maneiras, articulam-se com a plataformização da rede e contribuem para a construção de certas formas culturais e comunicacionais hegemônicas no mundo contemporâneo. Além disso, eles podem ser modificados sem intervenção humana direta e podem "tomar decisões" automatizadas, em certo sentido.

As consequências disso podem ser vistas na análise desenvolvida por Dodge e Kitchin sobre esses objetos: "Nós argumentamos que o software é idealizado para monitorar, administrar e processar informações sensiveis sobre as pessoas, os objetos e a interação entre eles, promovendo um novo tipo de governamentalidade que chamamos de gestão automatizada"3 (DODGE; KITCHIN, 2011, p. 85, tradução nossa).

\section{Ondas de Midiatização e Forças de Moldagem}

A partir dessas abordagens, pretendemos lidar, mesmo que parcialmente, com a hipótese desenvolvida por Couldry e Hepp (2017) de que nos deparamos com novas configurações comunicacionais e que o entendimento de seus fundamentos seria fundamental para a compreensão do processo da midiatização profunda do mundo contemporâneo.

Em suas análises sobre os processos de institucionalização de novas configurações comunicativas, Couldry e Hepp (2017) classificam as chamadas ondas de midiatização pelo tipo de força de moldagem que apresentam. A primeira, mecanizada (panfleto, livro, imprensa, fotografia, cinema); a segunda, eletroeletrônica (telégrafo, telefone, rádio e televisão); e, a terceira, digital (computador, internet, smartphones, objetos digitais). Como veremos, esta última, encontra-se com uma quarta onda, ou um desdobramento da sua força de moldagem digital, aquela que seria fundamental para o aprofundamento das formas de midiatização: a integração entre digitalização e dataficação.

Para os autores, o processo de dataficação, ou melhor, o encontro entre a terceira e a quarta onda de midiatização, poderia ser considerada a fase de midiatização profunda. Isso ocorreria devido à profunda incorporação dos media aos processos sociais mais básicos de nossa existência de um modo totalmente novo. Esses novos ambientes seriam configurados por redes de dispositivos conectados de diversas maneiras (media manifold) em meio aos quais seriam capturados, colonizados, apropriados os mais diversos modos de nossa existência.

2 Como veremos adiante, softwares "embarcados" são os objetos codificados (codjects), que dependem de códigos para iniciar ou executar tarefas de um dispositivo, mas que não podem ser considerados, exatamente, um computador.

3 No original: "We argue that software is ideally suited to monitoring, managing, and processing capta about people, objects, and their interaction, and is leading to a new mode of governmentality that we term automated management." 
A expressão utilizada por Sonia Livingstone $(2009,2011)$, que classifica a nossa época como a era da midiatização de tudo, seria um "sintoma" do modo pelo qual as novas configurações de nossas experiências comunicacionais poderiam ser formadas em um ambiente de midiatização profunda.

Como lidar, então, com esse adensamento tecnológico-comunicacional (MARTíN-BARBERO, 2003) dos tempos atuais? Em que sentido essa nova onda de midiatização seria "profunda" e diferente das outras?

A midiatização profunda seria, segundo os autores, uma matriz de configurações comunicativas apoiada na lógica das plataformas, objetos e ambientes digitais, que se integrariam a infraestruturas de registro e armazenamento de dados (data-driven infraestructures), capazes de combinar digitalização e dataficação em formas nunca vistas anteriormente: "Novas formas de interdependência estão emergindo aqui, baseadas não apenas na digitalização, mas na dataficação e na vinculação entre dataficação e categorização"5 (COULDRY; HEPP, 2017, p. 140, tradução nossa).

Dentro dessas novas forças de moldagem e dessas novas configurações comunicacionais, Andersen (2018) propõe que nos concentremos em três processos comunicativos fundamentais no mundo contemporâneo: os mecanismos de busca, os algoritmos e o uso de banco de dados. Posteriormente, vamos integrar a essa abordagem a presença fundamental dos objetos digitais, classificados, aqui, como objetos codificados de registro e compartilhamento de dados, os logjects.

\section{Plataformas, Cultura da Busca e Algoritmos}

Quando O'Reilly lançou o termo web 2.0, em 2005, para indicar o surgimento de uma nova configuração comunicacional da internet, muitos pesquisadores e pesquisadoras identificaram nesse novo ambiente as bases para a ampliação daquilo que foi chamado de cultura da participação. Entre suas bases conceituais, vamos destacar os trabalhos de Shirky (2011), Jenkins (2008) e Jenkins, Ford e Green (2014).

Algo que não estava muito claro na noção de web 2.0 era que o fato de que essas mudanças não estavam exatamente relacionadas aos aspectos interativos para o potencial criativo e colaborativo da cultura da participação, mas à identificação da própria Web como espaço de colaboração para desenvolvimento de plataformas de serviços: uma web programável, integrada por aplicativos (apps) e, consequentemente, por interfaces de programação de aplicações (APIs - Aplication Program Interface), como veremos adiante.

A internet, lentamente, deixava de ser vista como uma configuração comunicativa centrada em uma arquitetura de rede distribuída, aberta e colaborativa para se tornar um espaço de plataformização e, consequentemente, de desenvolvimento de aplicativos para essas plataformas ${ }^{6}$ :

Eu uso o termo plataformização para me referir à ascensão da plataforma como modelo infraestrutural e econômico dominante das redes sociais e às consequências de sua expansão para outras áreas de atividade online. Central para essa expansão é a utilização das APIs que transformam os sites de redes sociais em plataformas de mídia social... as plataformas facilitam a sua programabilidade para terceiros com o objetivo de descentralizar a produção de dados e centralizar a sua coleta (HELMOND, 2015, p. 5 , tradução nossa).

4 Como desenvolvemos em um artigo anterior, essa questão tem sido, também, uma preocupação crescente de Martín-Barbero na construção dos modelos de análise da teoria das mediações. A expressão que destacamos é utilizada por ele (GIRARDI JR, 2018).

5 No original: "New forms of interdependency are emerging here, based not on digitalization but on datafication, and the link of datafication to categorization".

6 Em sua investigação sobre música e geolocalização, Brotas (2017, p. 131) resgata a definição de software feita por Kitchin e Dodge no artigo "Code/Space: Software and Everyday Life": "a definição de software consiste em linhas de instruções em forma de códigos e algoritmos que, quando combinados, produzem rotinas e programas capazes de moldar funções digitais complexas. Ou, de forma um pouco mais simplificada, o software instrui o hardware (circuitos físicos e digitais, como discos rígidos, tocador de DVD, etc.) sobre o que fazer, "o que por sua vez pode gerar ação em outras máquinas, como ligar a energia elétrica, ligar um motor, ou fechar uma conexão" (KITCHIN; DODGE, 2011, p.20).

7 No original: "I use the term "platformization" to refer to the rise of the platform as the dominant infrastructural and economic model of the social web and the consequences of the expansion of social media platforms into other spaces online. Central to this is the offer of APIs, which turn social network sites into social media platforms... That is, platforms enact their programmability to decentralize data production and recentralize data collection". 
As plataformas são, antes de mais nada, sistemas programáveis ${ }^{8}$, sustentadas por diversas camadas (cabos de fibra ótica, datacenters, protocolos, códigos etc.) que funcionam basicamente como espaços de intermediação entre telecoms, big techs, produtores de conteúdo, fornecedores de serviços, anunciantes, consumidores e, um tipo muito particular de usuário: os desenvolvedores.

Esse processo de intermediação é, também, um processo-chave de controle sobre dados produzidos com propósitos muito específicos: busca (Google), padrões de deslocamento-tráfego (Uber), relacionamentos (Facebook), gosto musical (Spotify) etc. Em sua grande maioria, as plataformas são a estrutura para modelos multifacetados de negócios (SRNICEK, 2017). Elas são grandes armazenadoras de dados/metadados gerados pelos usuários, ao mesmo tempo em que, por meio dos desenvolvedores, são capazes de servir como recursos instrumentais de conexão entre a comunicação humana (que envolvem formatos reconhecidos por humanos - human-readable format) e os sistemas computacionais (que envolvem formatos reconhecidos por máquina - machine-readable format) (VAN DIJCK, 2017; VAN DIJCK; POELL; DE WALL, 2018).

A maior parte desses processos passa despercebida para a maioria das pessoas ou é vista, parcialmente, por um grupo, mais ou menos especializado, que atua em algumas áreas específicas relacionadas ao campo da computação.

Podemos dizer, com certeza, que a maioria dasforças de moldagem e suas configurações comunicativas sempre passaram por algum tipo de reificação?. A partir de um certo momento, no desenvolvimento dos dispositivos de comunicação, não se torna mais necessário conhecer os aspectos técnicos de seu funcionamento para produzir experiências simbólicas significativas a partir dele. No entanto, pretendemos mostrar como as configurações contemporâneas, que caracterizam a midiatização profunda, são de um tipo "novo" e, a compreensão disso, pode ser fundamental nas consequências que traz para o entendimento das experiências simbólicas estudadas em nosso campo.

Pela lógica das plataformas, toda vez que os usuários se conectam com objetos e dispositivos digitais, eles precisam ser submetidos a um tratamento de de-composição e re-composição, ou seja, as ações humanas, que produzem cotidianamente, precisam ser traduzidas em dados legiveis por máquina. Essa é a condição necessária para que aspectos da subjetividade e das práticas humanas possam ser submetidas a processos de automação e gestão em larga escala (dataficação).

As decisões tomadas pelas plataformas sobre essas questões acabam gerando padrões culturais, pois, elas passam a se enredar nas próprias práticas comunicacionais, interferindo nos processos de representação e de construção da subjetividade dos próprios usuários desses sistemas (ROUVROY; BERNS, 2015, GILLESPIE, 2018a; SILVEIRA, 2019). Os processos de subjetivação não ocorrem à margem das configurações comunicacionais. Eles são parte de um mesmo processo.

Logo, uma das consequências dessa nova configuração é que uma boa parte dos processos humanos, particularmente, aqueles relacionados a critérios de relevância e autoridade - sobre aquilo que deve ser considerado ou não na tomada de decisões - passa a ser mediado por uma lógica algorítmica (GILLESPIE, 2018a).

O que está em jogo, é o modo pelo qual as plataformas definem "quem somos nós", não a partir de uma razão metafísica qualquer, mas a partir de seus propósitos específicos como plataformas. Elas não estão fazendo um simples ajuste entre interesses e desejos dos usuários, como uma espécie de mão invisivel, entre oferta e demanda cultural, centrada na automação e maximização do processo. O que é feito, antes de mais nada, é a adequação desses interesses e desejos aos propósitos e às ofertas disponíveis pelas plataformas (ROUVROY; BERNS, 2015; DAUBS; MANZEROLLE, 2015).

8 A “programabilidade" seria, justamente, a característica principal das plataformas (HELMOND, 2015).

9 Ao falar de reificação, Hepp (2014) observa, ainda, que além de configurar experiências de institucionalização, as forças de moldagem tendem a se ocultar como aparatos técnicos. 
Elas podem parecer um simples processo de automatismo preditivo, uma vez que, o usuário sequer precisa gerar uma busca para encontrar o que "precisa", ela the é recomendada. No entanto, não seria inadequado identificá-las como processos de colonização de nossas práticas textuais, que se equilibram sobre uma nova forma de economia política comunicacional: "A co-construção complexa entre os algoritmos e os usuários e entre a utilidade da plataforma e a geração de lucro é um ato de equilíbrio intricado em que as affordances tecnológicas e sociais [...] desempenham um papel importante"10 (BILLIC, 2016, p.3, tradução nossa).

Quando o próprio Google torna pública as regras de indexação que utiliza, a plataforma não está apenas nos ajudando a otimizar o acesso a artigos, bens ou serviços que procuramos ou que temos a oferecer. Seu sistema modula um estilo, um tipo de gênero textual, otimizado segundo suas próprias regras algorítmicas de identificação de autoridade e relevância em seu sistema de recomendação, preditivo $0^{11}$. Como esses algoritmos não funcionam de forma eficiente sem que estejam integrados a gigantescos bancos de dados, todo o processo de consolidação das plataformas passa por uma espécie de "acumulação primitiva de dados" dentro de modelagens desenvolvidas para esses mesmos algoritmos (STRIPHAS, 2015; ARAÚJO, 2017; SRNICEK, 2017; GROHMANN, 2020)

Nos processos de midiatização profunda, precisamos romper com certa reificação, construída pelos porta-vozes dessas plataformas que as apresentam como portadoras de uma pretensa objetividade algorítmica. Precisamos romper, também, com um determinado regime de verdade digital e enfatizar que não existem algoritmos "enviesados", uma vez que, não existem critérios universais ou estritamente "técnicos" de determinação da relevância das regras e valores que utilizamos em nossas condutas e escolhas (BEER, 2016; BREITER; HEPP, 2018).

Podemos fazer a mesma observação sobre a expressão "dados brutos". Gillespie (2018b), apoiado em uma advertência de Gitelman (2013) sobre o tema, observa que os dados submetidos à lógica algorítmica precisam de algum modo de "preparo", alguma regra de categorização. Nesse sentido, Beer $(2010,2013,2017)$, Cheney-Lippold (2011, 2017), Orton-Johnson e Prior (2013), Striphas (2015) e Gillespie (2018b), Berry e Fagerjord (2017), entre outros, já destacaram a importância do desenvolvimento de uma espécie de sociologia crítica dos algoritmos para enfrentarmos as questões trazidas pela integração entre a camada cultural e a camada computacional em nossas vidas no mundo contemporâneo (SILVA, 2020; GIRARDI JR., 2017). Van Dijck traduz, também, de modo bem claro, a necessidade desse tipo de crítica:

As pilhas de (meta)dados são propositalmente geradas a partir de diferentes plataformas on-line que são tudo menos objetivas [...] A compreensão dos padrões requer, portanto, avaliação crítica: por que nós buscamos certos padrões nas pilhas de metadados, o que interessa e com quais objetivos? Identificar padrões significativos a partir dos dados retirados de plataformas on-line é um ato intrinsecamente interpretativo, ainda que as prerrogativas implícitas sejam explicitadas (VAN DIJCK, 2017, p. 47).

Além disso, as configurações comunicacionais de um ambiente concentrado em plataformas são marcadas por um profundo paradoxo: elas se apresentam como igualitárias, neutras, públicas, com vínculos locais, horizontalizadas em termos de poder (ou em um modelo bottom-up), mas, concomitantemente,

10 No original: "The complex co-construction between the algorithms and users and between the utility of the platform and profit generation is an intricate balancing act where both technological and social affordances (Postigo, 2016) play an important role."

11 Castro (2019, p.6), resgata a noção de interpelação recursiva, desenvolvida por Manovich (2001): "Na interpelação recursiva que se verifica nas plataformas algorítmicas, podemos identificar a ideia de movimento na atividade do usuário, a partir da qual são obtidos dados sobre ele, e as ideias de destino e de retorno à origem na interpelação propriamente dita, customizada para cada usuário com base naqueles dados. Também a ideia de risco está arraigada na governança algorítmica, visto que, ao prever comportamentos, busca transformar a incerteza em risco a ser gerenciado". Lemos e Bitencourt (2018), por sua vez, destacam a formação de uma nova sensibilidade performativa: "Esses objetos sentem o mundo, produzem dados e agem de forma autônoma e independente de uma intervenção humana direta". Esse processo é dinâmico e depende de uma complexa interrelação entre as apropriações dos usos das plataformas e os dispositivos digitais em sua conexão com a lógica algorítmica e o modelo econômico que impulsiona o seu desenvolvimento. 
funcionam como sistemas hierarquizados de controle privado e comercial, marcado por decisões econômicas, ideológicas e políticas (top-down) de impacto global (VAN DIJCK, 2017; VAN DIJCK; POELL; DE WALL, 2018).

As Ciências Sociais e as Ciências da Comunicação produziram reflexões suficientes para nos afastar dessa visão positivista e tecnocrática da suposta objetividade algorítmica, funcionando como estabilizadora de confiança. Uma postura na qual somos tentados a cair, justamente, devido a determinados tipos de reificação (BILLIC', 2016; SILVEIRA, 2017; D'ANDREA; JURNO; DALBEN, 2019; SILVA, 2020).

\title{
Os logjects e a midiatização profunda do cotidiano
}

A partir da hipótese levantada por Andersen (2018), a Cultura da Busca e a Cultura do Algoritmo, orientada por dados, seriam parte da configuração comunicacional na qual se apoiam os processos de midiatização profunda. No entanto, essa hipótese não seria completa, sem uma análise do lugar dos dispositivos digitais e dos objetos codificados de registro e de compartilhamento de dados (logjects) nesse processo.

Segundo Hesmondhalgh e Meier (2018), o mundo ocidental, no final do século XX, viu um complexo deslocamento e integração entre a indústria de eletrodomésticos e as indústrias de tecnologia da informação, produzindo um novo tipo de mídia e, consequentemente, novas experiências de uso e apropriações desses objetos. Essa integração pode ser vista no desenvolvimento de dispositivos digitais (de uso doméstico ou móveis) como o Walkman, Discman, iPod e os smartphones e smart TVs.

Podemos dizer que o conceito que orientou o desenvolvimento dos smartphones e as smart TVs representou o que poderia ser chamado de dispositivo total, capaz de integrar todas as funcionalidades de múltiplos dispositivos por meio do download de aplicativos (apps) compativeis com seus sistemas operacionais.

Como boa parte das experiências cotidianas passou a ser integrada à lógica das plataformas, os dispositivos smart tornaram-se elementos fundamentais de registro e indexação das informações sobre os comportamentos dos seus usuários. Esse processo tornou-se ainda mais profundo com o desenvolvimento das app stores (MATVIYENKO, 2014).

Os dispositivos smart não são representados apenas pelos smartphones. Eles são parte de uma nova configuração comunicacional que abarca, também, aquilo que poderíamos chamar de tecnologias domésticas. Estamos diante de um tipo particular de integração entre plataformas, dispositivos e objetos digitais:

\begin{abstract}
[...] mais detalhadamente, exploramos a relação entre software, objetos e a materialidade espacial, examinando como a incorporação de microprocessadores e algoritmos nos objetos que as pessoas usam para realizar suas atividades domésticas acabam por transformá-los, embutindo-os da capacidade de produzir tipos novos e adicionais de atividade no mundo como parte de uma formação ator-rede diversa [...] Os objetos, como vamos explicar, estão ganhando competências adicionais, tais como: monitorar o ambiente, registrar sua própria atividade, assumir alguns tipos de decisões para seus donos[...]12 (DODGE; KITCHIN, 2009, p. 1344, tradução nossa).
\end{abstract}

Quanto mais esses objetos se aproximam da condição de logjects ${ }^{13}$, mais são capazes de criar a configuração comunicacional necessária para os processos de aprofundamento da midiatização.

12 No original: "[...] we more fully explore the relationship between software, objects, and material spatiality, examining how the embedding of microprocessors and software algorithms into the objects people use to undertake daily domestic tasks is transforming these objects, imbuing them with capacities that allow them to do additional and new types of work in the world as part of diverse actant networks. [...] Objects, as we will illustrate, are gaining additional competencies: to sense their environment, to record their own use, to take over aspects of decision making from their human owners $[. .]$.$" .$

13 Optamos pela valorização do termo objetos codificados de registro e compartilhamento de dados (logjects), embora seu uso não seja dominante no meio acadêmico, devido à ausência de outro termo adequado, capaz de traduzir a natureza desses tipos de objetos digitais, que, em nossa abordagem, seriam fundamentais para o aprofundamento da midiatização no mundo contemporâneo. Além de Dodge e Kitchin (2009), o termo serve de base para uma série de autores que consideramos relevantes nos estudos sobre Sociologia Digital como Beer (2010, 2013), Bleecker (2011), Burrows e Beer (2013). O seu uso em pesquisas, em língua portuguesa, só foi encontrado em Brotas (2017). 
Não existiria tão intensa captura de dados para as plataformas se não fosse a disponibilidade constante de acesso aos dispositivos digitais, que carregam sistemas operacionais (softwares) de registro e compartilhamento de dados, além de suportarem a instalação e o funcionamento de variados tipos de logjects: os aplicativos.

É uma tendência que está sendo ampliada, consideravelmente, com a expansão desses objetos codificados de registro e compartilhamento de dados por dispositivos eletroeletrônicos, reconfigurando o ambiente doméstico (BIHR; THORNE, 2016). Portanto, se há algo de "profundo" ou de "adensamento tecnológico-comunicacional" nesse processo, entendemos que ele passa pelo desenvolvimento dos logjects e os diferentes modos pelos quais integram os dispositivos digitais, permitindo a conexão constante com as plataformas e a cultura algorítmica orientada por dados. No desenvolvimento dessa hipótese, vamos identificar os diferentes tipos de objetos digitais que podemos encontrar no mundo contemporâneo.

Dodge e Kitchin (2011) chamam nossa atenção para dois tipos específicos de objetos digitais que, cada vez mais, podem ser encontrados em nosso cotidiano. Os primeiros são os objetos em que o código não tem uma função essencial para o funcionamento de um dispositivo. Eles são os objetos codificados perifericamente (peripherally coded objects), que podemos encontrar nos conhecidos medidores digitais de velocidade/distância de bicicletas e de tempo de cozimento em um forno. Na maioria das vezes, eles servem de apoio a dispositivos analógicos.

O segundo tipo de objeto digital é aquele que têm o que chamamos de software embarcado, ou seja, eles são os objetos codificados (codjects), que dependem de códigos para iniciar ou executar tarefas de um dispositivo, mas que não podem ser considerados, exatamente, um computador.

Os objetos codificados [codejects] podem ser subdivididos, por sua vez, em três principais classes baseadas nas seguintes características: sua programabilidade, interatividade, capacidade para "recordar", "habilidade" para ações preditivas a partir de padrões prévios de uso e de sua capacidade relacional (conectiva). Em resumo, são classificados em objetos codificados rígidos, objetos codificados unitários e objetos codificados de registro e compartilhamento de dados ${ }^{14}$ (DODGE; KITCHIN, 2011 p. 54, tradução nossa, grifos nossos).

Vamos desenvolver as características desses três tipos de objetos codificados:

1) os objetos codificados rígidos (hard codejects) são baseados em códigos, têm um software rodando em seu microprocessador, mas não são programáveis. É o caso dos dispositivos USB (Universal Serial Bus),

2) Os objetos codificados unitários (unitary coded objects - UCOs), são baseados em códigos e permitem algum tipo de programação - o que faz com que os usuários possam controlar algumas das suas funções. São chamados de unitários porque tem tudo o que precisam para funcionar em si mesmos. Eles podem ser divididos em objetos codificados unitários fechados, que não usam dados do mundo exterior (relógios digitais, DVD players) e objetos codificados unitários com sensores, que reagem às variações do ambiente em que se encontram (encontram-se no ar-condicionado, máquinas de lavar, câmeras digitais, por exemplo)

3) os objetos codificados de registro e compartilhamento de dados (logjects), que concentram nossa maior atenção e serão definidos adiante.

14 No original: "Codejects can be further subdivided into three main classes on the basis of the following characteristics: their programmability, interactivity, capacity for remembering, their ability for anticipatory action in the future based on previous use, and relational capacities. In summary, these classes are hard codejects, unitary codejects, and logjects". 
Como ilustração desse sistema de classificação, Dodge e Kitchin apresentam o seguinte fluxograma para identificação desses tipos de objetos:

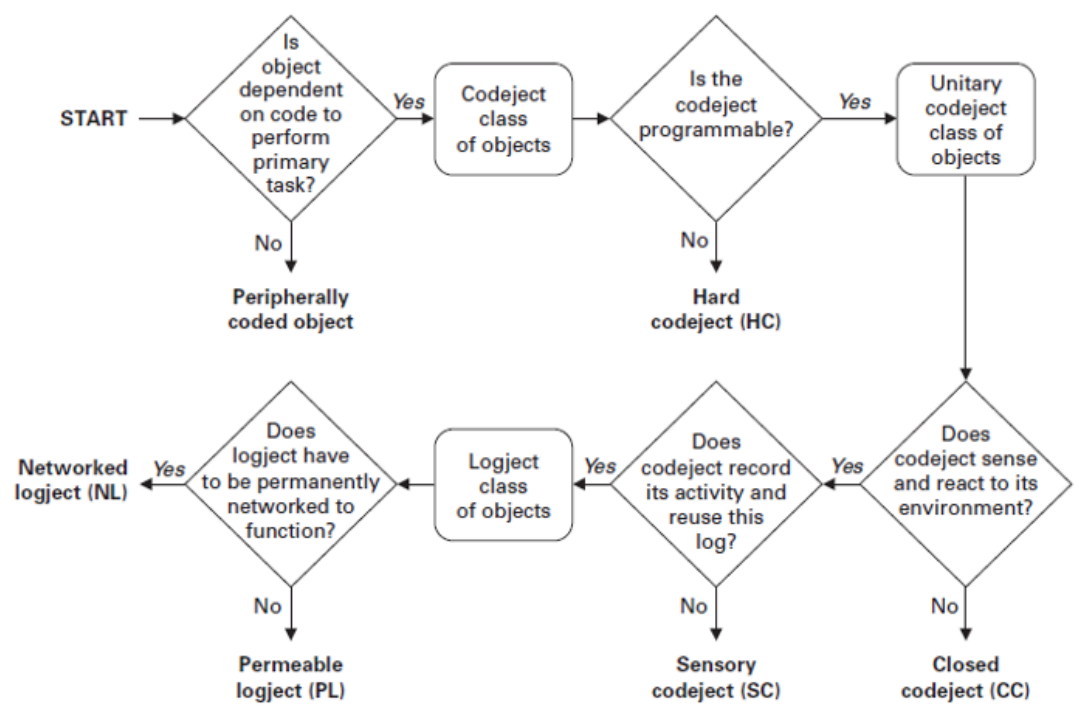

Figura 01: Quadro de instruções para identificação dos tipos de objetos digitais encontradas no livro Code/Space de Dodge e Kitchin (2011, p. 55)

Fonte: Dodge e Kitchin (2011)

Para a análise das forças de moldagem da midiatização profunda, os objetos que nos interessam, aqui, são os do terceiro tipo: os logjects. Eles são os objetos digitais que registram sua atividade, mesmo quando desligados, desenvolvendo um tipo de "percepção" de si mesmos e do modo pelo qual são usados (awareness of themselves ou aware of how they are being used) e podem se conectar, ou não, a complexas redes de outros objetos, trocando dados com eles. Os logjects:

[...] exibem vivacidade - um sentimento de que as possibilidades de sua exploração são infinitas; plasticidade - as pessoas que interagem com o codject têm a sensação de poder levá-los ao seu limite de uso sem danificá-los; incrementação - seus aspectos computacionais melhoram e evoluem com o uso, e, disrupção - a computação embarcada é aberta a usos imprevisiveis e pode reagir a isso sem travamentos (Murtaugh 2008) ${ }^{15}$ (DODGE; KITCHIN, 2011, p. 56, tradução nossa, grifos nossos).

Eles seriam uma nova classe de objetos emergentes que geram registros de seu próprio uso no tempo e no espaço e podem compartilhar essa informação em rede, produzindo "decisões" e ações autônomas e automatizadas ${ }^{16}$. São capazes de produzir dados e metadados indexáveis, tornam possíveis trocas dessas informações com outros logjects e são capazes de estabelecer diversas formas de conexão entre máquina-máquina, máquina-humano, humano-humano.

15 No original: "They, along with logjects, exhibit liveness - a feeling that there are infinite possibilities to explore; plasticity - the person interacting with the codeject feels that they can push its limits without breaking the system; accretion - the computation improves and evolves with use; and interruption - computation is open to unpredictable input and can react to it without breakdown (Murtaugh 2008)." O uso do realce foi uma decisão que tomamos, pois os termos parecem estar deslocados pelo autor.

16 Este termo é adaptado de blogject, utilizado por Bleecker (2011), em um artigo intitulado: “Por que as coisas importam: um manifesto sobre objetos em rede". O termo blog é integrado a object para ressaltar o aspecto de disseminação de informações sobre sua existência que o próprio objeto gera. Ele seria o equivalente ao que Dodge e Kitchin chamam de objetos codificados de registro e compartilhamento de dados em rede (networked codject) 
Os logjects são uma evolução dos spimes, ou seja, objetos que carregavam, de modo integrado, sistemas de identificação e geolocalização por meio de identificadores de radiofrequência, conhecidos como RFID (SPEED, GIANNI, SHINGLETON, 2010). A complexidade dos spimes residia na combinação de recursos de Global Positioning System (GPS) e de Local Positioning System (Bluetooth, Wi-Fi, RFID) naquilo que se convencionou chamar de serviços baseados em geolocalização (Located-Based Services). O seu desenvolvimento gerou dois tipos particulares de logjects: os impermeáveis e os permeáveis.

Os logjects impermeáveis são aqueles que registram dados, iniciam tarefas automaticamente, são capazes de realizar troca de dados localizados com outros logjects, mas não se conectam a uma rede. Os primeiros robôs aspiradores poderiam ser considerados um exemplo desse tipo.

Os logjects considerados permeáveis são aqueles que registram dados de seu histórico de uso e integram-se a uma complexa rede de conexões com outros dispositivos. Em alguns casos, eles dependem dessa conexão para realizarem suas funções e é, por meio dela, que registram e monitoram centenas de atividades rotineiras, permitindo o desenvolvimento da cultura da busca, do algoritmo e da cultura orientada por dados.

Podemos destacar, como exemplo, os sistemas operacionais dos dispositivos móveis (IOS e Android) ${ }^{17}$, os wearables, os assistentes pessoais (PDA - Personal Digital Assistent), os apps de streaming (Deezer, Sportify, Netflix, Amazon Prime Video etc), os apps baseados em monitoramento e gestão de serviços ligados a geolocalização (Google Maps, Waze etc.), que podem ser integrados aos apps de streaming (a autorização de uso do Spotify no interior da plataforma Waze e o, consequente, compartilhamento de dados) e um conjunto de apps instalados em dispositivos domésticos (smart TVs, streaming boxes, etc.).

Os apps, abreviação de aplicativos, são pacotes simplificados de software que podem ser integrados a diversos tipos de dispositivos digitais devido à compatibilidade que possuem com os seus sistemas operacionais. Por meio desse processo, eles são capazes de funcionar como interfaces para operações realizadas naquilo que vem sendo chamado de nuvem (cloud) ${ }^{18}$, ou seja, eles funcionam como uma interface muito particular entre um espaço de dados e os dispositivos digitais de uso pessoal (smartphones, wearables, smart TVs). Segundo Bratton (2014), sua instalação pode ser feita em plataformas específicas como as App Stores e seu uso é destinado a finalidades muito específicas (checar e postar mensagens, comparar preços e fazer compras, corrigir imagens, ver e recomendar filmes, checar multas etc.).

Consideramos as contribuições de Dodge e Kitchin (2011) fundamentais para explorarmos essa nova classe de objetos emergentes e o modo pelo qual estariam no centro dos processos de midiatização profunda:

Refletindo sobre essa taxonomia e os modos pelos quais os objetos estão se tornando, externamente, legíveis por máquina, ao mesmo tempo em que se tornam, internamente, codificados, vemos que a natureza de muitos desses objetos e processos materiais, que constituem a nossa vida cotidiana, estão sendo reformulados de modo bastante radical - os objetos estão sendo reconfigurados e definidos de um modo alternativo, ganhando capacidades para realizar atividades adicionais no mundo, ao mesmo tempo em que o mundo passa a produzir mais trabalho neles e por meio deles. Agora, esses

\footnotetext{
17 “Os apps requerem "[...] certas especificações dos sistemas operacionais de modo a funcionar de modo apropriado. 0 sistema operacional faz a mediação entre o hardware e os apps por meio de um conjunto de interfaces, que permitem aos aplicativos rodar em dispositivos com diferentes configurações de hardware." (MATVIYENKO, 2014, p. xviii, tradução nossa). No original: "[...] It requires certain operating-system specifications in order to function properly, The operating system mediates between hardware and apps by means of the set of system interfaces, which enables apps to run on the devices with different hardware capabilities."

18 “Diversas coisas devem ser ditas sobre a nuvem. Em primeiro lugar, ela é um sistema operacional - de modo mais preciso, ela é o Sistema Operacional da Internet (IOS), que roda como um software de acesso a informação em rede e, portanto, permite aos usuários mover a informação dos datacenters para dispositivos computacionais quase que instantaneamente." (MATVIYENKO, 2014, p.xix, tradução nossa). No original: Several things have to be said about the cloud. First of all, it is an operating system - more precisely, the Internet Operating System (IOS) - that runs as a Web browser and, therefore, allows users to move information from the datacenters onto computational devices almost instantaneously.
} 
objetos são percebidos de novas maneiras - eles são identificáveis individualmente e seu uso e sua mobilidade são rastreáveis no tempo e no espaço. ${ }^{19}$ (DODGE; KITCHIN, 2011, p.59-60, tradução nossa).

\section{Considerações finais}

Couldry e Hepp (2017) definem a era da midiatização profunda contemporânea pela integração entre uma terceira e quarta onda de midiatização e seus processos de moldagem, ou seja, uma configuração midiática centrada nos processos de digitalização e de dataficação. De certo modo, podemos dizer que todas essas ondas, suas forças de moldagem e configurações comunicativas geraram midiatizações profundas de algum tipo e, a própria noção de profundo, só pode ser avaliada historicamente.

Nesse sentido, procuramos mostrar que o aprofundamento ou adensamento dos processos de midiatização assume uma forma muito particular no mundo contemporâneo e é marcado pela convergência e integração, intensa e extensivamente, entre os processos culturais (human-readable) e a lógica dos processos computacionais (machine-readable).

Os ambientes digitais em que construímos as experiências de nós mesmos no mundo são de uma natureza diferente. Entendê-los, torna-se uma tarefa fundamental em nosso campo. Cada vez mais, eles são capazes de registrar dados, reconhecer padrões, propor ou, até mesmo, realizar de forma automatizada certas "tomadas de decisão". Eles não apenas registram informações sobre sua relação com os seres humanos, mas, compartilham esses registros com outros objetos, identificando padrões algorítmicos em uma complexa rede de conexões.

Ao buscar uma maior clareza sobre a "profundidade" dessa nova forma de midiatização, integramos à hipótese de Couldry e Hepp (2017), particularmente, as reflexões de Andersen (2017, 2018). Trouxemos, para isso, suas posições sobre os processos de plataformização, sobre a construção de padrões que caracterizariam uma cultura da busca, baseada em lógica algorítmica, e o significado de uma cultura orientada por dados.

Berry e Fagerjord (2017) nos alertam para as consequências dessa virada computacional (computational turn), particularmente, no que diz respeito ao tipo de questões que precisamos formular na intersecção entre os estudos em ciências humanas, em ciências da comunicação e da informação.

Nesse sentido, trouxemos, ainda, as análises de Gillespie (2017, 2018a, 2018b), Cheney-Lippold (2011, 2017), Striphas (2015), Rouvroy e Berns (2015), entre outros, que destacam o modo pelo qual as essas plataformas assumem uma forma cultural. Isso pode ser visto na discussão sobre os critérios de relevância e de autoridade algorítmica dos sistemas de busca e recomendação, subordinados aos processos de comodificação de dados, que se acentuam quanto mais nos integramos a esses novos ambientes, produzindo uma nova governamentalidade algorítimica.

Dodge e Kitchin $(2005,2009,2011)$, por sua vez, mostraram como a criação e a presença dos logjects em nosso cotidiano é um elemento fundamental no aprofundamento dos processos de digitalização, plataformização e de dataficação.

19 "No original: Reflecting on this taxonomy, and the ways in which objects are becoming either externally machine-readable or endogenously coded, it seems to us that the nature of many objects and the material processes that constitute everyday life are being remade in quite radical ways - objects are being alternatively reconfigured and defined, they are gaining additional capacities to do additional work in the world, and the world can do more work on and through them. Individual objects are now knowable in new ways - they are uniquely identifiable and their use and movement is trackable across space and time. 
No momento da tradução para o português e a publicação de A Construção Mediada da Realidade, em 2020, procuramos explorar um campo de possibilidades de investigação, centrado na noção de midiatização profunda, que manteria essa proposta de abordagem no centro de um debate sobre a comunicação no mundo contemporâneo. Com certeza, essa reflexão terá que se desdobrar em investigações teóricas e empíricas sobre as diversas formas e escalas em meio às quais esses processos podem ser articulados historicamente. 


\section{Referências}

AMPUJA, Marco; KOIVISTO, Juha; VÄLIVERRONEN, Esa. Strong and Weak Forms of Mediatization Theory. A Critical Review. NORDICOM Review, v. 35 (Special Issue), p. 111-123, 2014.

ANDERSEN, Jack. Search, searching, search engines: new media interactions in digital culture. Informationcultures: Big data - small meaning - global discourses. mar 6, 2017, Disponivel em: $<$ https://medium.com/big-data-small-meaning-and-globaldiscourses/search-searching-search-enginesnew-media-interactions-in-digital-culturebd1775bf8d6d>. Acesso em: 25 fev. 2018.

. Archiving, ordering, and searching: search engines, algorithms, databases, and deep mediatization. Media, Culture \& Society, v. 40, n. 8, p. 1135-1150, 2018.

ARAÚJO, Willian F. Os Algoritmos da Comunicação: uma abordagem de processos computacionais como objeto de estudo da comunicação. In: CONGRESSO BRASILEIRO DE CIÊNCIAS DA COMUNICAÇÃO (INTERCOM), 40., 2017, Curitiba. Anais... Curitiba: Intercom, 2017.

BEER, David. Mobile Music, Coded Objects and Everyday Spaces. Mobilities, v. 5, n. 4, p. 469-484, 2010.

Popular Culture and New Media: the politics of circulation. London: Palgrave Macmillan, 2013.

The social power of algorithms. Information, Communication \& Society, v. 20, n. 1, p. 1-13, 2017.

BEER, David; BURROWS, Roger. Popular Culture, Digital Archives and the New Social Life of Data. Theory, Culture \& Society, v. 30, n. 4, p. 47-71, 2013.

BERRY, David. The computational turn: thinking about the digital humanities. Culture Machine, v. 12, p. 1-22, 2011. .; FAGERJORD, Anders. Digital Humanities. Cambridge/UK: Polity Press, 2017.

BIHR, Peter; THORNE, Michelle. Understanding the Connected Home. 2.ed. [S.l.]: Kindle Edition, 2016.

BILLIC', Pasko. Search algorithms, hidden labour and information control. Big Data \& Society, v. 3, n. 1, p. 1-9, jan./jun. 2016.

BLEECKER, Julian. Remaking Everyday Objects. In: KITCHIN, Rob; DODGE, Martin. CODE/SPACE: software and everyday life. Cambridge/MA: MIT Press, 2011.

BRAGA, José Luiz. Mediatização como Processo Interacional de Referência. Animus, Santa Maria, RS, v. 5, n. 2, p. 9-35, jul./dez. 2006.

BRATTON, Benjamin H. On Apps and Elementary Forms of Interfacial Life: Object, Image, Superimposition. In: MILLER, Paul D.; MATVIYENKO, Svitlana. The imaginary App. Massachusetts: MIT Press, 2014. 
BREITER, Andreas, HEPP, Andreas. The Complexity of Datafication: Putting Digital Traces in Context. In: HEPP, Andreas, BREITER, Andreas, HASEBRINK, Uwe (Eds.). Communicative Figurations. London: Palgrave Macmillan, 2018.

BROTAS, Diego. Música, mídia e espacialidades: reapropriações do lugar para o desenvolvimento de relações musicais (geo)localizadas. 2017. 227 f. Tese (Doutorado em Comunicação) - Instituto de Artes e Comunicação Social, Universidade Federal Fluminense, Niterói, 2017.

CARVALHO, Carlos Alberto. MIDIATIZAÇÃO: investigações brasileiras e europeias e o midiacentrismo. In: ENCONTRO ANUAL DA COMPÓS, 25., 2016, Goiânia. Anais... Goiânia: Compós, 2016.

CASTRO, Julio Cesar L. de. Plataformas Algorítmicas: interpelação, perfilamento e performatividade. Famecos, Porto Alegre, v. 26, n. 3, p. 1-24, set./dez. 2019.

CHENEY-LIPPOLD, John. A new Algorithmic Identity - soft biopolitics and the modulation of control. Theory, Culture \& Society, v. 28, n. 6, p. 164-181, 2011.

. We are Data: algorithms and the making of our digital selves. New York: New York University Press, 2017.

COULDRY, Nick. Mediatization or Mediation? Alternative Understandings of the Emergent Space of Digital Storytelling. New Media and Society. 10(3), p. 373-391, 2008.

COULDRY, Nick; HEPP, Andreas. Conceptualizing Mediatization: Contexts, Traditions, Arguments.

Communication Theory, v. 23, p. 191- 202, 2013.

The Mediated Construction of Reality. Cambridge/UK: Polity Press, 2017.

A Construção Mediada da Realidade. São Leopoldo/RS: Editora Unisinos, 2020.

DAUBS, Michael; MANZEROLLE, Vincent. App-centric mobile media and commoditization:Implications for the future of the open Web. Mobile Media \& Communication, v. 4, n. 1, jul. 2015.

DODGE, Martin; KITCHIN, Rob. Codes of life: identification codes and the machine-readable World.

Environment and Planning D: Society and Space, v. 23, p. 851-881, 2005.

Software, Objects, and Home Space. Environment and Planning A: Economy and Space, v. 41, n. 6 , 1344-1365, 2009.

Code/Space. Cambridge, MA: The MIT Press, 2011.

FAUSTO NETO, A. Midiatização, prática social - prática de sentido. In: ENCONTRO ANUAL DA COMPÓS, 15., 2006, Bauru. Anais... Bauru: Compós, 2006.

FERREIRA, Jairo. Midiatização: dispositivos, processos sociais e de comunicação. E-Compós, Brasília, v. 10, 2007. 
FERREIRA, Jairo et al. (Orgs). Entre o que se diz e o que se pensa: onde está a midiatização? Santa Maria: FACOS/UFSM, 2018.

FINNEMANN, Niel O. Mediatization Theory and Digital Media. Communications: the European Journal of Communication Research, v. 1, n. 36, p. 67-89, 2011.

FIGUEIRAS, R. Estudos em Mediatização: causalidade, centralidade, interdisciplinaridade. São Paulo. Matrizes, v. 11, n. 1, p. 101-126, 2017

GILLESPIE, Tarleton. The politics of "platforms". New Media \&Society, v. 12, p. 347-364, 2010.

. A Relevância dos Algoritmos. Parágrafo, São Paulo, Brasil, v. 6, n. 1, p. 95-121, jan./abr. 2018a.

. Platforms are not Intermediaries. Georgetown Law Technology Review, v.2, n. 2, p. 198-214, 2018b.

GIRARDI JR., Liráucio. O Estranho mundo da informação - e da materialidade - no campo da comunicação. E-Compós, Brasília, v. 20, n.1, p.1-18, jan./abr. 2017.

De Mediações em Mediações: a questão da tecnicidade em Martín-Barbero. Matrizes, São Paulo, v. 12, n. 1, p. 155-172, jan./abr. 2018.

GITELMAN, Lisa (Ed.). Raw data is an oxymoron. Cambridge, MA: MIT Press, 2013.

GOMES, Pedro Gilberto. Midiatização: um conceito, múltiplas vozes. Famecos, Porto Alegre, v. 23, n. 2, 2016.

GROHMANN, Raphael. Plataformização do trabalho: entre a dataficação, a financeirização e a racionalidade neoliberal. Eptic, v. 2, n. 1, p. 107-122, jan./abr. 2020.

; MAURO, Rosana. O Potencial Teórico do Conceito de Midiatização e os Estudos sobre Classes Sociais na Comunicação. Novos Olhares, v. 4, n. 1, p. 246-257, jun. 2015.

GUNKEL, D. J. Beyond mediation: thinking the computer otherwise. Interactions, Bristol, v. l, n. 1, p. 53-70, 2009.

HELMOND, Anne. The Platformization of the Web: Making Web Data Platform Ready. Social Media + Society, v.1, n. 2, p. 1-11, 2015.

HESMONDHALGH, David; MEIER, Leslie M. What the digitalisation of music tells us about capitalism, culture and the power of the information technology sector. Information, Communication \& Society, v. 21, n. 11, p. 1555-1570, 2018.

HEPP, Andreas. As configurações comunicativas de mundos midiatizados: pesquisa da midiatização na era da "mediação de tudo". MATRIZes, v. 8, n. 1, p. 45-64, jan./jun. 2014.

; HJARVARD, Sig.; LUNDBY, Knut. Mediatization: theorizing the interplay between media, culture and society media. Culture \& Society, London, v. 37, n. 2, p. 314-324, 2015. 
; HASEBRINK, Uwe. Interação Humana e Configurações Comunicativas: transformações culturais e sociedades midiatizadas. Parágrafo, v. 2, n. 3 p. 75-89, dez. 2015.

HJARVARD, Stig. Midiatização: teorizando a mídia como agente de mudança social e cultural. Matrizes, ano 5, n. 2, p. 53-91, jan./jun. 2012.

Da Mediação à Midiatização: a institucionalização das novas mídias. Parágrafo, v. 2, n. 3, p. 51-62, jul./dez. 2015.

JANOTTI Jr., Jeder, MATOS, Ângela, JACKS, Nilda. (Orgs). Mediação \& Midiatização.

Brasília: Compós; Salvador: EDUFBA, 2012.

JENKINS, Henry. Cultura da Convergência. São Paulo: Aleph, 2008.

; FORD, Sam; GREEN, Joshua. Cultura da Conexão: criando valor e significado por meio da mídia propagável. São Paulo: Aleph, 2014.

JOHNSON, Steven. Cultura da Interface. Rio de Janeiro: Jorge Zahar Editor, 2001.

LEMOS, André; BITTENCOURT, Elias. Sensibilidade performativa e comunicação das coisas. São Paulo.

Matrizes, v.12, n. 3, p. 165-188, set./dez. 2018

LIVINGSTONE, Sonia M. On the mediation of everything. Journal of Communication, v. 59, n. 1, p. 1-18, 2009.

If Everything is Mediated, What is Distinctive About the Field of Communication? International

Journal of Communication, v. 5, p. 1472-1475, 2011.

MANOVICH, Lev. The Language of New Media.Cambridge/Mass: MIT Press, 2001

MARTÍN-BARBERO, Jésus. Pistas para entre-ver meios e mediações. In:

. Dos meios às mediações:

comunicação, cultura e hegemonia. Rio de Janeiro: Editora UFRJ, 2003. p. 11-21.

MARTINO, Luís Mauro Sá. Rumo a uma teoria da midiatização: exercício conceitual e metodológico de sistematização. Intexto, Porto Alegre, n. 45, p. 16-34, mai./ago. 2019.

MATVIYENKO, Svitlana. Introduction. In: MILLER, Paul D.; MATVIYENKO, Svitlana. The imaginary App. Massachusetts: MIT Press, 2014.

MINTZ, André Goes. Midiatização e plataformização: aproximações. Novos Olhares, v. 8, n. 2, p. 98-109, 2019.

ORTON-JOHNSON, Kate, PRIOR, Nick (eds). Digital Sociology: critical perspectives. Londres: Palgrave Macmillan, 2013.

ROUVROY, Antoinette; BERNS, Thomas. Governamentalidade algorítmica e perspectivas de emancipação: o díspar como condição de individuação pela relação. Revista Eco-Pós, v. 18, n. 2, p. 36-56, 2015. 
RÜDIGER, Francisco. Os estudos de mídia e a problemática epistemológica da teoria da midiatização uma nova escolástica? Intexto, Porto Alegre, n. 34, p. 745-769, set./dez. 2015.

SHIRKY, Clay. A Cultura da Participação. São Paulo: Zahar, 2011.

SILVA, Tarcizio. Racismo Algorítmico em plataformas digitais: microagressões e discriminação em código. In: (Org.). Comunidades, algoritmos e ativismos digitais: Olhares afrodiaspóricos. São Paulo: LiteraRUA, 2020.

SILVEIRA, Sérgio Amadeu. Governo dos Algoritmos. Revista de Políticas Públicas, v. 21, n. 1, p. 267-281, 2017. A noção de modulação e os sistemas algorítmicos. Revista de Comunicação da FAPCOM, São Paulo, v. 3, n. 6, p. 31-46, nov. 2019.

SRNICEK, Nick. Plataform Capitalism. Cambridge/UK: Polity Press, 2017.

SODRÉ, Muniz. Antropológica do espelho: uma teoria da comunicação linear e em rede. Petrópolis, RJ: Vozes, 2002.

SPEED, Chris; GIANNI, Corino; SHINGLETON, Duncan. 'Pervasive Memory, Locative Narratives', REM Research on Education and Media, v. 2, n. 2, p. 40-47, 2010.

STRIPHAS, Ted. Algorithmic Culture. European Journal of Cultural Studies, v. 18, n. 4-5, p. 395-412, 2015.

VAN DIJCK, José. Confiamos nos dados? As implicações da datificação para o monitoramento social. MATRIZes, São Paulo, v.11, n. 1, p. 39-59, jan./abr. 2017.

VAN DIJCK, Jose; POELL, Thomas; DE WALL, Martijn. The Plataform Society: public values in a connective world. New York: Oxford University Press, 2018.

VERÓN, Eliseo. Teoria da midiatização: uma perspectiva semioantropológica e algumas de suas consequências. MATRIZes, v. 8, n. 1, p. 13-19, 2014. 


\section{Informações sobre 0 artigo}

\section{Resultado de projeto de pesquisa, de dissertação, tese}

Humanidades Digitais e Comunicação de Interesse Público.

\section{Fontes de financiamento}

Não se aplica.

\section{Considerações éticas}

Não se aplica.

\section{Declaração de conflito de interesses}

Não se aplica.

\section{Apresentação anterior}

Revisão do artigo: "Deep Mediatization": rumo a uma outra comunicação?

42o Congresso Brasileiro de Ciências da Comunicação - Intercom

\section{Agradecimentos/Contribuições adicionais}

Meus agradecimentos aos membros do GP Teorias da Comunicação da Intercom, à Comissão Editorial da E-Compós e aos pareceristas pelo rigor na análise e pelas contribuições sugeridas. Os limites do artigo, no entanto, são de minha inteira responsabilidade. 